Canadian

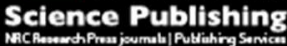

Biochemistry and Cell Biology

Biochimie et biologie cellulaire

\title{
Adaptations to Excess Choline in Insulin Resistant and Pcyt2 Deficient Skeletal Muscle
}

\begin{tabular}{|r|l|}
\hline Journal: & Biochemistry and Cell Biology \\
\hline Manuscript ID & bcb-2016-0105.R1 \\
\hline Manuscript Type: & Article \\
\hline Date Submitted by the Author: & $29-J u l-2016$ \\
\hline Keyword: List of Authors: & $\begin{array}{l}\text { Taylor, Adrian; University of Guelph College of Biological Science, Human } \\
\text { Health and Nutritional Sciences } \\
\text { Schenkel, Laila; University of Guelph College of Biological Science, Human } \\
\text { Health and Nutritional Sciences } \\
\text { Yokich, Maiya; University of Guelph College of Biological Science, Human } \\
\text { Health and Nutritional Sciences } \\
\text { Bakovic, Marica; University of Guelph, }\end{array}$ \\
\hline &
\end{tabular}


3 Adrian Taylor, Laila Cigana Schenkel, Maiya Yokich and Marica Bakovic*

4 Department of Human Health and Nutritional Sciences, University of Guelph, Guelph, Ontario, 5 Canada

6

$11 *$ Corresponding author

12 University of Guelph, ANNU Bldg Rm 346, Guelph, Ontario, Canada N1G 2W1

13 Telephone: $519-824-4120 \times 53764$

14 Fax: 519-763-5902

15 Email: mbakovic@uoguelph.ca

16

17

18 


\section{Abstract}

It was hypothesized that choline supplementation in insulin resistant (IR) CTP:phosphoethanolamine cytidylyltransferase deficient $\left(\mathrm{Pcyt}^{+/-}\right)$mice would ameliorate muscle function by remodeling glucose and fatty acid (FA) metabolism. Pcyt $2^{+/-}$mice either received no treatment or were allowed access to $2 \mathrm{mg} / \mathrm{ml}$ choline in drinking water for 4 weeks. Skeletal muscle was harvested from choline treated and untreated mice. Lipid analysis and metabolic gene expression and signaling pathways were compared between untreated Pcyt $2^{+/-}$ mice, treated Pcyt $2^{+/-}$mice and Pcyt $2^{+/+}$mice. The major positive effect of choline supplementation on IR muscle was the reduction of glucose utilization for FA and TAG synthesis and increased muscle glucose storage as glycogen. Choline reduced the expression of genes for FA and TAG formation (Scd1, Fas, Srebp1c, Dgat1/2), upregulated the genes for FA oxidation (Cpt1, Ppar $\alpha$, Pgc1 $\alpha$ ) and had minor effects on phospholipid and lipolysis genes. Pcyt $2^{+/-}$muscle had reduced insulin signaling (IRS1), autophagy (LC3) and choline transport (CTL1) proteins that were restored by choline treatment. Additionally, choline activated AMPK and Akt while inhibiting mTORC1 phosphorylation. These data established that choline supplementation could restore muscle glucose metabolism by reducing lipogenesis and improving mitochondrial and intracellular signaling for protein and energy metabolism in insulin resistant Pcyt2 deficient mice.

Key words: Pcyt2, choline, skeletal muscle, insulin resistance, triglycerides

Abbreviations: Pcyt2-CTP:phosphoethanolamine cytidylyltransferase; CEPT-choline ethanolamine phosphotransferase 1; PE-phosphatidylethanolamine; FA-fatty acid; CHO-choline treated; PC-phosphatidylcholine; ACh-acetylcholine; TAG-triglyceride; IR-insulin resistance; PI 
41 phosphatidylinositol; FC-free cholesterol; PC-phosphatidylcholine; CE-cholesterol ester; DAG-

42 diacylglycerol; CTL1-choline transporter like protein 1; Pcyt1-CTP:phosphocholine

43 cytidylyltransferase; $P s s 1$ and $P s s 2$-phosphatidylserine synthase 1 and 2; Psd-phosphatidylserine

44 decarboxylase; Srebpl-sterol regulatory binding protein 1; Fas-fatty acid synthase; Scd1-

45 stearoyl-CoA desaturase 1; Dgat1 and Dgat2-diacylglycerol acyltransferase 1 and 2; Lpl-

46 lipoprotein lipase; $H s l$-hormone-sensitive lipase; Atgl-adipocyte triglyceride lipase; Ppar $\alpha$ -

47 peroxisomal proliferation activation receptor $\alpha$; Pgcl $\alpha$-Ppar-gamma coactivator $1 \alpha$; Acc-acyl

48 CoA carboxylase; Cpt1-carnitine palmitoyltransferase 1; Gapdh-glyceraldehyde-3-phosphate

49 dehydrogenase 
Introduction

51 The nutrient choline is required for the formation of membrane phospholipids

52 phosphatidylcholine (PC) and sphingomyelin, the neurotransmitter acetylcholine (ACh)

53 (Niculescu 2013) and the methyl group donor betaine (Zeisel 2012). Choline is a vital

54 component of skeletal muscle function and choline deficiency is directly implicated in the

55 development of muscular dystrophy (Sher et al. 2006). Choline specific transporter

56 CTL1/SLC44A1 is abundantly present in skeletal muscle (Yuan et al. 2004) and upregulated in

57 numerous mitochondrial and muscle myopathies (Yuan et al. 2006). Inherited mutations in

58 choline kinase beta result in the development of muscular dystrophy in both mouse and humans

59 (Sher et al. 2006 and Mitsuhashi et al. 2011). ACh is essential for muscle contraction (Yamada et

al. 2012) and both neuron-specific (Haga 1971) and ubiquitous choline transporters (Michel and

61 Bakovic, 2012) supply choline for muscle ACh synthesis (Nassenstein et al. 2015). Choline

62 deficiency compromises muscle cell function by decreasing mitochondrial abundance, increasing

63 TAG accumulation and perturbing phospholipid and FA metabolism (Michel et al. 2011).

64 The ATTICA study (Detopoulou et al. 2008) as well as the Nurses' Health Study (Zeisel and da

65 Costa 2009) showed that diets rich in choline and betaine could reduce inflammatory biomarkers,

66 including C-reactive protein, interleukin-6 and tumor necrosis factor alpha. Adequate intakes of

67 choline and betaine have been frequently associated with lower plasma homocysteine, an

68 independent risk factor for cardiovascular disease. In experimental animals as well as humans,

69 choline deficiency results in the formation of non-alcoholic fatty liver and an increased

70 sensitivity to inflammation (Tryndyak et al. 2016). Choline and betaine are named lipotropic

71 dietary agents because they can prevent or reduce the accumulation of TAG (fat) in the liver. 
72 Betaine has been shown to curb liver fat accumulation and it has been broadly recognized as a

73 safe and effective therapy for alcoholic fatty liver disease (Purohit et al. 2007). The beneficial

74 role of choline and betaine was also clearly observed with respect to non-alcoholic

75 steatohepatitis (which develops in obesity) when oral supplements improved homocysteine

76 levels, lipid profile (attenuation of fatty liver and proper export and formation of triglycerides

77 and VLDL), steatosis, inflammation and fibrosis (Cordero et al. 2013). Therefore, it is clear that

78 a diet enriched in choline is beneficial in the prevention and treatment of obesity related

79 disorders. However, it is not known if dietary choline could regulate muscle function and

80 mitigate muscle IR and lipotoxicity.

81 It is becoming increasingly evident that skeletal muscle phospholipids are important mediators of 82 insulin sensitivity and muscle contraction. Muscle-specific knockout models for 83 CTP:phosphoethanolamine cytidylyltransferase/Pcyt2 (Selathurai et al. 2015) and 84 choline/ethanolamine phosphotransferase 1/CEPT1 (Funai et al. 2016) have been recently 85 developed. These enzymes catalyze the rate-regulatory step and the final step respectively in the 86 CDP-ethanolamine (Kennedy) pathway for phosphatidylethanolamine (PE) biosynthesis. The 87 two knockout models established that impaired PE biosynthesis has a direct impact on muscle 88 lipid metabolism and function (Selathurai et al. 2015 and Funai et al. 2016). The muscle-specific 89 knockout (Selathurai et al. 2015) accumulated diacylglycerol (DAG) and TAG but did not 90 develop insulin resistance (IR), probably because of upregulated mitochondrial FA oxidation.

91 CEPT1-deleted muscle (Funai et al. 2016) was on the other hand protected from high-fat diet IR

92 but was exercise intolerant, which has been attributed to PE dependent regulation of endoplasmic 93 reticulum calcium handling. We have generated a heterozygous Pcyt2 mouse (Pcyt $2^{+/-}$) which 
94 has systemically reduced PE biosynthesis (Basu et al. 2015). Young Pcyt2 $2^{+/-}$mice are

95

96

97

asymptomatic but gradually develop IR, obesity and metabolic syndrome, including the accumulation of DAG and TAG in skeletal muscle (Fullerton et al. 2007 and Schenkel et al. 2015). Choline treatment reduced Pcyt $2^{+/-}$obesity and fatty liver disease (Schenkel and Bakovic 2014). Here, we hypothesise that choline will also aid in mitigating Pcyt $2^{+/-}$muscle pathologies by restoring FA and general energy homeostasis, altogether resulting in improved muscle insulin signalling and mitochondrial function.

\section{Methods}

\section{Animals and choline treatments}

Generation of Pcyt2 deficient $\left(\mathrm{Pcyt}^{+/-}\right)$mice and genotyping were described previously (Basu et al. 2015). All procedures were approved by the University of Guelph's Animal Care Committee and were in accordance with guidelines of the Canadian Council on Animal Care. Mice were exposed to a 12-h light/12-h dark cycle beginning with light at 7:00 a.m. Mice were fed a standardized chow diet (Harlan Teklad S-2335) ad libitum and had free access to water. Pcyt $2^{+/-}$ mice progressively develop obesity and insulin resistance starting at 2.5 months of age (Fullerton et al. 2007). In the present study, 8-10 month old mice are divided into three groups $(\mathrm{n}=6-10$ in each group): Pcyt $2^{+/-}$treated $\left(\mathrm{Pcyt} 2^{+/-} \mathrm{CHO}\right)$, and untreated $\left(\mathrm{Pcyt} 2^{+/-}\right)$with choline, and wildtype littermate controls $\left(\mathrm{Pcyt}_{2}^{+/+}\right)$. Treated mice were kept in separate cages and had free access to water containing $2 \mathrm{mg} / \mathrm{ml}$ choline (Sigma) for 4 weeks. Choline supplementation did not influence food and water intake, as determined on a daily basis. The choline intake from drinking water was $\sim 240 \mu \mathrm{g} / \mathrm{g} /$ day as determined from the choline concentration $(2 \mathrm{mg} / \mathrm{ml})$, water intake 
116 (5 ml/day/mouse), and mouse weight (Schenkel et al. 2015). Thus, in addition to $17 \mathrm{mg} / \mathrm{day}$

117 obtained from diet, the Pcyt $2^{+/-} \mathrm{CHO}$ group obtained additional $10 \mathrm{mg}$ choline/day from water, 118 which was a 59\% increase in total choline intake. The recommended adequate intake (AI) for 119 choline is $425 \mathrm{mg}$ /day for women and $550 \mathrm{mg} /$ day for men (Penry and Manore 2008).

121 Analysis of phospholipids and cholesterol

122 The analysis of muscle phospholipids and cholesterol was performed by HPLC (Agilent 123 Technologies, Santa Clara, CA, USA) as before (Schenkel et al. 2015). Briefly, 1 mg of skeletal 124 muscle homogenates from Pcyt $2^{+/-} \mathrm{CHO}, \mathrm{Pcyt}^{+/-}$and Pcyt $2^{+/+}$mice $(\mathrm{n}=3)$ was extracted in the 125 presence of an internal standard (50 mg dipalmitoyl-phosphatidyl dimethyl ethanolamine) using 126 a method of Folch et al (1957). The extracted lipids were dried, resuspended in 127 chloroform:isooctane (1:1) and separated by HPLC using a 3-solvent gradient and a normal 128 phase column (Onyxmonolithic silica; Phenomenex Incorporated, Torrance, CA, USA). The 129 amounts of PC, PE, phosphatidylinositol (PI), sphingomyelin (SM), cholesterol (FC), cholesterol 130 ester (CE) and free fatty acids (FA) were determined using appropriate standards and expressed 131 in $\mathrm{mg}$ of lipid/mg of protein.

134 Muscle samples (50 mg, $\mathrm{n}=3$ per group) were homogenized in $200 \mu 15 \%$ PBS-T, the 135 homogenates twice heated to $100^{\circ} \mathrm{C}$ and cooled to room temperature and centrifuged to remove 136 protein debris. Total TAG content ( $\mu \mathrm{g} / \mathrm{mg}$ of muscle) was determined using Wako Diagnostics 137 Kit. In addition, $50 \mathrm{mg}$ muscle samples ( $\mathrm{n}=3$ per group) were extracted by the method of Bligh 138 and Dyer (Bligh and Dyer 1959). The lower organic phase was dried and resuspended in $100 \mu \mathrm{l}$ 
139 of chloroform. DAG and TAG were resolved by TLC using a solvent system of 140 heptane/isopropyl ether/acetic acid (60:40:3). Authentic standards were spotted in parallel to the 141 samples, the plates were dried and exposed to iodine vapour for visualization. DAG and TAG 142 spots were scanned and quantified with densitometry (Fullerton et al. 2007).

144 Analysis of muscle glycogen

145 Skeletal muscle samples (50 mg; $\mathrm{n}=3$ per group) were hydrolyzed in $500 \mu 130 \% \mathrm{KOH}$. 146 Homogenates were heated to $100^{\circ} \mathrm{C}$ for $30 \mathrm{~min}$ with frequent vortexing. Glycogen was 147 precipitated with $600 \mu \mathrm{l}$ of $95 \%$ ethanol, kept on ice for $45 \mathrm{~min}$ and centrifuged at $840 \times \mathrm{g}$ for 30 $148 \mathrm{~min}$. Glycogen pellet was dissolved in $1 \mathrm{ml}$ of water by vortexing. The glycogen solution was 149 mixed with $1 \mathrm{ml}$ of $5 \%$ phenol and $5 \mathrm{ml}$ of $98 \%$ sulfuric acid was then added and kept at room 150 temperature for $10 \mathrm{~min}$. Samples were cooled on ice for $30 \mathrm{~min}$ and developed colour which was 151 determined spectrophotometrically at $490 \mathrm{~nm}$. A standard curve was generated using pure 152 glycogen (Roche) (Lo et al. 1970).

154 Immunoblotting

155 Muscle samples (50 mg; $\mathrm{n}=3$ per group) were homogenized in cold lysis buffer (10 mM Tris$156 \mathrm{HCl}[\mathrm{pH} 7.4], 1 \mathrm{mM}$ EDTA and $10 \mathrm{mM} \mathrm{NaF})$ containing protease $(1 / 10)$ and phosphatase 157 (1/100) inhibitor cocktails (Sigma). The lysates were centrifuged for 20 minutes at 10,000 x g at $1584^{\circ} \mathrm{C}$ to remove cell debris and $50 \mu \mathrm{g}$ of lysate was used for analysis. For CTL1 protein, the 159 lysates were mixed with a non-denaturing loading buffer (62 mM Tris-HCl and $0.01 \%$ 160 bromophenol blue in 10\% glycerol) and resolved with an $8 \%$ native gel at $90 \mathrm{~V}$ for $2 \mathrm{~h}$. All other 
161 proteins were assessed under denaturing conditions with a loading buffer comprised of $400 \mathrm{mM}$

162 Tris-HCl, 50\% glycerol, 10\% SDS, 0.25\% (w/v) bromophenol blue and 3\% (w/v) DTT. AMPK $\alpha$, 163 pAMPK $\alpha$, ACC, pACC, PKCa, PI3K, IRS-1, Akt, p ${ }^{308}$ Akt, p p ${ }^{473}$ kt, mTORC1 and $164 \mathrm{p}^{2443}$ mTORC1 were resolved with a 10\% denaturing gel. LC3I and LC3II were resolved with a $16515 \%$ denaturing gel. All proteins were transferred to PVDF membranes (VWR). Membranes 166 were blocked with 5\% skim milk in PBS-T and incubated with the C-terminal CTL1 antibody 167 (ENS-627; 1:500). Membranes were also incubated with AMPK $\alpha$, pAMPKa, PI3K, Akt, $168 \mathrm{p}^{308} \mathrm{Akt}, \mathrm{p}^{473} \mathrm{Akt}, \mathrm{mTORC1}$ and $\mathrm{p}^{2443} \mathrm{mTORC1}$ (Cell Signaling), LC3 I/II (Abcam), IRS-1 169 (Millipore) antibodies as a 1:1,000 dilution with 5\% BSA in PBS-T overnight at $4^{\circ} \mathrm{C}$. 170 Membranes were washed 2 times with PBS-T and incubated with horseradish peroxidase171 conjugated anti-rabbit IgG secondary antibody (NEB) (1:5,000) in 5\% skim milk in PBS-T for 1 172 h. After washing with PBS-T, the proteins were visualized using a chemiluminescent substrate 173 (Sigma). $\beta$-tubulin (Sigma) was used as a loading control.

176 mRNA was extracted from $\mathrm{Pcyt}^{+/-} \mathrm{CHO}, \mathrm{Pcyt}^{+/-}$and $\mathrm{Pcyt} 2^{+/+}$skeletal muscle $(\mathrm{n}=5-8$ per 177 group) using TRIzol reagent (Invitrogen). cDNA was prepared from $2 \mu \mathrm{g}$ of total mRNA using a 178 3-AP primer and SuperScript II reverse transcriptase (Invitrogen). For the phospholipid 179 pathways, the expression of the choline transporter like protein 1 (Ctll), CTP:phosphocholine 180 cytidylyltransferase (Pcyt1), phosphatidylserine synthase 1 and 2 (Pss1 and Pss2) and 181 phosphatidylserine decarboxylase $(P s d)$ were analyzed. Genes involved in FA and TAG 182 synthesis included sterol regulatory binding protein 1c (Srebp1c), FA synthase (Fas), stearoyl- 
183 CoA desaturase 1 (Scd1), and diacylglycerol acyltransferase 1 and 2 (Dgat1 and Dgat2). The 184 expression of the lipid degradation genes lipoprotein lipase $(L p l)$, hormone-sensitive lipase $(H s l)$ 185 and adipocyte triglyceride lipase $(A t g l)$ was also analyzed. The expression of the mitochondrial 186 regulatory genes peroxisomal proliferation activation receptor $\alpha$ (Ppar $\alpha$ ), Ppar gamma 187 coactivator $1 \alpha(P g c l \alpha)$, and the FA genes acyl CoA carboxylase $(A c c)$ and carnitine 188 palmitoyltransferase $1(C p t 1)$ were used to assess mitochondrial FA metabolism. 189 Glyceraldehyde-3-phosphate dehydrogenase (Gapdh) mRNA was used as a control. The primers 190 and PCR conditions are in Table 1 and as described (Basu et al. 2015, Schenkel et al. 2015, 191 Schenkel and Bakovic 2014).

193 Statistical analysis

194 Data are represented as mean \pm SEM. One way ANOVA with post-hoc Tukey's test was 195 performed to compare means between WT, Pcyt $2^{+/-}$and Pcyt $2^{+/-}$CHO groups. A difference at $19695 \%$ confidence interval $(\mathrm{p} \leq 0.05)$ was considered as significant. All statistical tests were 197 performed with GraphPad Prism 4 software.

198

199 Results

200 Choline modifies membrane composition and reduces TAG accumulation in Pcyt $2^{+-}$muscle

201 The analysis of the membrane lipids in choline treated and control muscle is shown in Fig. 1A.

202 The content of the major phospholipids PE and PC in the Pcyt $2^{+/-}$muscle was similar to the 203 content in the control Pcyt $2^{+/+}$muscle. The IR Pcyt $2^{+/-}$muscle however had reduced levels of the 
204 cell signalling phosphatidylinositol (PI) and the 'lipid raft' free cholesterol (FC). Choline 205 treatments $\left(\mathrm{Pcyt}^{+/-} \mathrm{CHO}\right)$ did not improve the PI content but significantly increased PC, 206 sphingomyelin (SM) and FC, reaching the levels present in the control muscle. Additionally, free 207 FA content was dramatically $(60 \%)$ reduced by choline treatment (Fig. 1A). Overall, choline 208 treatment increased the membrane phospholipid ratio (PC/PE, Fig. 1B) but did not improve the 209 "lipid rafts' ratio (SM/FC, Fig. 1C). The levels of Pcyt $2^{+/-}$muscle TAG were elevated relative to control muscle, and became 211 significantly reduced by choline treatment. Pcyt $2^{+/-}$muscle had $60 \%$ (Fig. 1D) more TAG than 212 the control muscle and choline reduced muscle TAG by $40 \%$. A similar trend was observed in 213 TAG and DAG relative ratios (Fig. 1E); Pcyt $2^{+/-}$muscle DAG was elevated by $30 \%$ relative to 214 control Pcyt $2^{+/+}$muscle and choline $\left(\mathrm{Pcyt}^{+/-} \mathrm{CHO}\right)$ returned the elevated Pcyt2 $2^{+/-}$DAG to the 215 control levels. Taken together, the lipid analysis established that the major positive effects of 216 choline diet on the IR Pcyt $2^{+/-}$muscle were the increased quantities of the choline-containing 217 membrane phospholipids (PC and SM) and cholesterol and the reduced quantities of the energy 218 producing FA, DAG and TAG.

221 Choline treatment had a positive effect regarding the restoration of Pcyt $2^{+/-}$muscle glycogen 222 levels (Fig. 1F). Glycogen was reduced in Pcyt $2^{+/-}$muscle in relation to Pcyt $2^{+/+}$wildtype muscle 223 by $50 \%$. One month of dietary choline supplementation was able to increase Pcyt $2^{+/-}$muscle 224 glycogen levels by $60 \%(\mathrm{p}<0.05)$. Taken together, the data in Figures $1 \mathrm{~A}-\mathrm{F}$ demonstrated that 
225 choline supplementation dramatically remodelled Pcyt2 ${ }^{+/}$muscle TAG and glycogen metabolism to be more similar to the lean phenotype of Pcyt $2^{+/+}$muscle.

\section{Choline does not modify phospholipid gene expression}

229 Choline is incorporated into PC by the CDP-choline (Kennedy) pathway. Choline transporter 230 Ctll and the main regulatory gene for PC synthesis Pcyt1 did not differ among the three groups.

231 The expression of PsS1, which catalyzes the degradation of PC and the formation of PS from PC, 232 was decreased by $15 \%(\mathrm{p}<0.05)$ with choline treatment. This was also observed with Pss 2 , 233 which catalyzes the formation of PS from PE $(20 \%$ reduction, $\mathrm{p}<0.05)$. The expression of $P s d$, 234 which catalyzes the mitochondrial formation of PE from PS, was also not altered with choline 235 treatments and it was similar across the three groups (Fig. 2A). Taken together, these data 236 demonstrated that dietary choline did not significantly modify the genes for phospholipid 237 synthesis in muscle but had a modest reducing effect on PC and PE metabolic genes.

Choline down-regulates Pcyt $2^{+/}$muscle lipogenesis

240 Since choline treatment reduced FA, DAG and TAG levels in muscle of obese mice (Fig.1. A, D 241 and E), we then investigated the effect of choline on the expression of genes involved in FA and 242 TAG synthesis (Fig. 2B). The main regulators of lipogenesis, Srebplc, and its target genes Fas 243 and $S c d 1$ were elevated in $P c y t 2^{+/-}$relative to Pcyt $2^{+/+}$control muscle and they were reduced by 244 choline treatment $(25 \%, 15 \%$ and $15 \%$ respectively, $\mathrm{p}<0.05)$, to become similar to the 245 expression observed in the wildtype muscle. The expression of Dgat1 and Dgat2, which catalyze 
246 the formation of TAG from FA and DAG, were elevated in $\mathrm{Pcyt}^{+/-}$relative to Pcyt $2^{+/+}$muscle

247 and both decreased with choline treatment $(20 \%$ and $35 \%$ respectively, $\mathrm{p}<0.05)$. Reduced

248 muscle lipolysis is linked to IR and obesity (Kase et al. 2015). Choline supplementation

249 modestly increased the expression of $\mathrm{Lpl}(10 \%, \mathrm{p}<0.05) . H s l$ and adipose triglyceride lipase

$250(A \operatorname{tgl})$ were only slightly different from wildtype muscle and unaffected by choline treatments.

251 Taken together, the gene expression data and the metabolic analysis in Fig. 1 established that in

252 the IR Pcyt $2^{+/-}$muscle, choline supplementation primarily reduced de novo FA synthesis and 253 lipogenesis while having a minor effect on DAG/TAG degradation by lipolysis.

Choline improves AMPK activity and FA oxidation in Pcyt $2^{+-}$muscle

256 As shown in Fig. 3A and 3C, AMPK activity (pAMPK:AMPK) was dramatically reduced in 257 Pcyt $^{+/-}$muscle relative to the wildtype muscle $(3$ fold, $\mathrm{p}<0.05)$. AMPK activity was 258 significantly increased ( 2 fold) by choline treatment, indicating improvements in Pcyt $2^{+/-}$ 259 mitochondrial energy production. Additionally, ACC phosphorylation (pACC:ACC) which was 260 diminished in Pcyt $2^{+/-}$mice $(2$ fold, $\mathrm{p}<0.05)$ was increased with choline supplementation $(2$ 261 fold, $\mathrm{p}<0.05)$, indicating that choline inhibited the first committed step in de novo FA synthesis 262 (Fig. 3B and 3C). In addition to decreased FA synthesis and increased pAMPK activity, there 263 was an increase in FA oxidation with choline treatment. The expression of genes involved in FA 264 oxidation - Ppara, Cpt1 and Pgcl $\alpha$ (Fig. 3D) were reduced $20-35 \%$ in Pcyt $2^{+/-}$muscle relative to $265 \mathrm{Pcyt}^{+/+}$control muscle. Choline $\left(\mathrm{Pcyt} 2^{+/-} \mathrm{CHO}\right)$ stimulated their expression by $20-25 \%$, back to 266 the levels observed in the control muscle. ACC mRNA expression was similar between the 
267 groups, however the inhibition of ACC activity through increased phosphorylation (AMPK 268 activation) is described in Fig. 3A-C.

271 To support improvements in FA formation (Fig. 2) and mitochondrial oxidation (Fig 3), we then 272 assessed the effect of choline on the insulin signaling pathway. As shown in Fig. 4A, the most 273 dramatic effect of choline was the restoration of IRS1 protein levels and Akt phosphorylation 274 (Fig. 4B-E). The amount of IRS1 in $\mathrm{Pcyt}^{+/-}$muscle was diminished by $80 \%$ and choline 275 recovered IRS1 protein to the control levels (Fig. 4B). Downstream from IRS1, the most affected 276 was the mTORC2 regulated phosphorylation of Akt at $\operatorname{Ser}^{473}$ ( $p^{473}$ Akt:Akt). Pcyt2 $2^{+/-} p^{473}$ Akt was 277 reduced $50 \%$ and choline increased $\mathrm{p}^{473}$ Akt levels 3 fold (Fig. 4E). Interestingly, Akt 278 phosphorylation at $\mathrm{Thr}^{308}\left(\mathrm{p}^{308} \mathrm{Akt}\right.$ :Akt, Fig. 4D) was not modified by Pcyt2 deficiency or 279 affected by choline treatment. Finally, choline dramatically reduced (50\%) the amount of PKC $\alpha$ 280 (Fig. 4F), the well-established inhibitor of muscle insulin signalling (Mellor and Parker 1998).

281 Because choline improved Pcyt2 $2^{+/-}$muscle AMPK activity and insulin signaling, we also probed 282 the regulation of mTORC1 (protein synthesis) and LC3 (autophagy), downstream of both AMPK 283 and insulin signaling (Fig. 5A-D). Indeed, there was a 60\% increase in mTORC1 284 phosphorylation at $\operatorname{Ser}^{2448}$ in $\mathrm{Pcyt}^{+/-}$muscle with respect to wildtype muscle, and choline 285 completely prevented this increase in phosphorylation ( $\mathrm{p}^{2448} \mathrm{mTORC1}: \mathrm{mTORC} 1$ in Fig 5B). 286 Since mTORC1 is a negative regulator of autophagy, we also probed the level of lipidation of the 287 autophagy marker LC3 (LC3II:LC3I in Fig 5C). The lipidation of LC3 was reduced in Pcyt2 $2^{+/-}$ 
288 muscle and choline increased LC3 lipidation to the level present in the control muscle. As there 289 are alterations in autophagy occurring with choline treatment, it was also of interest to determine 290 the amount of the choline transporter CTL1, previously shown to be degraded by the 291 autophagosomal-lysosomal pathway in palmitate treated muscle cells (Schmitz-Peiffer 2002).

292 The CTL1 protein as well as the total LC3 protein were however dramatically reduced (by $70 \%$ ) 293 in Pcyt $^{+/-}$muscle and almost completely recovered by choline treatment, implying elevated 294 proteosomal degradation in Pcyt2 ${ }^{+/}$muscle (Fig. 5D). Taken together, the above data established 295 that choline was able to restore not only insulin signalling but also protein synthesis and turnover 296 in Pcyt2 ${ }^{+/}$muscle. 
297

298

299

300

301

302

303

304

305

306

307

308

309

310

311

312

313

314

315

316

317

318

\section{Discussion}

This study assessed the effects of dietary choline on IR Pcyt $2^{+/-}$muscle. Choline decreased muscle TAG accumulation by reducing expression of FA and TAG biosynthetic genes and by increasing mitochondrial genes for energy utilization. Choline raised muscle glycogen levels and restored the activity of the metabolic regulators AMPK, Akt and mTORC1. Overall, choline improved glucose and lipid balance and insulin signaling in IR Pcyt $2^{+/-}$muscle.

One-month of choline supplementation in Pcyt $2^{+/-}$mice increased the content of cholinecontaining phospholipids (PC and SM) and cholesterol (FC). This increased the membrane phospholipid ratio but did not modify the lipid raft ratio in the skeletal muscle. The major effect of choline supplementation was the reduction in FA, DAG and TAG content that were otherwise elevated in the IR Pcyt $2^{+/-}$muscle. Based on previous studies (Kase et al. 2015), one may anticipate that choline facilitated TAG degradation by lipolysis, which was not the case. Choline mediated effects were primarily via reduction in FA synthesis by lipogenesis and FA esterification/TAG formation regulated by Srebp1c and Dgat1/2 respectively. The expression of Srebpl and its target genes Fas and Scd1 as well as Dgat1/2 were significantly reduced while the lipolysis genes Lpl and Atgl were not affected by choline treatment. Srebp1 is positively regulated by insulin signaling and downstream mTORC1 but it is not known how it became upregulated in IR states to cause elevated lipogenesis and an accumulation of TAG in the liver and perhaps other tissues. On the other hand, it has been established that AMPK mediated mTORC1 inhibition decreases the ability for Srebp1c to localize to the nucleus in IR skeletal muscle (Liu et al. 2012). Here we established that together with impaired insulin signaling, mTORC1 was upregulated and AMPK was down regulated in IR Pcyt $2^{+/-}$muscle. Choline 
319 supplementation improved insulin signaling, upregulated AMPK and downregulated mTORC1, consequently reducing the expression of Srebp1c and lipogenesis.

We previously established by NMR metabolomic studies that the majority of choline 322 supplied to $\mathrm{Pcyt}^{+/-}$mice became oxidized to betaine, which enters the one-carbon cycle and 323 stimulates mitochondrial oxidation. The increased flux through the one-carbon cycle prevents the 324 accumulation of choline and betaine in the circulation (Schenkel et al. 2015). Plasma acyl325 carnitine composition after choline supplementation to $\mathrm{Pcyt} 2^{+/-}$mice further supports this 326 possibility (Schenkel et al. 2015). Therefore, the main positive effect of choline on Pcyt $2^{+/-}$ 327 muscle could be attributed to its action as a methyl group donor, and subsequently stimulating 328 mitochondrial FA oxidation. High dietary choline and betaine intakes are significantly associated 329 with favorable body composition in humans (Gao et al. 2016) and Sachan et al. 2005 330 demonstrated that choline supplementation lowers oxidative stress during exercise. Recently, 600 $331 \mathrm{mg}$ per day of alpha glycerylphosphorylcholine has been recommended to supplement the diets 332 of speed and power athletes to enhance their muscle performance (Bellar et al. 2015). In 333 addition, betaine has been used as an ergogenic reagent to improve muscle performance and to 334 increase body mass (Cholewa et al. 2014), presumably by increasing circulating IGF levels 335 leading to increased PI3K/Akt signaling (Senesi et al. 2013). More recently, it has been 336 demonstrated that betaine supplementation reduces obesity and systemic IR by upregulation of 337 Fgf21 (Ejaz et al. 2016). In addition to the effects on muscle FA oxidation through the betaine/one-carbon cycle, 339 choline could have influenced acetylcholine signaling, which is essential for muscle contraction. Reduction in acetylcholine production has a marked influence on muscle performance and most 
341 sport nutrition products are supplemented with choline to maintain acetylcholine levels (Penry 342 and Manore 2008). The mechanism of muscle contraction after acetylcholine stimulation 343 primarily depends on AMPK activation and is independent of insulin (Koh et al. 2008). IR is 344 associated with muscle weakness and exercise ameliorates IR via AMPK activation and $\mathrm{Ca}^{2+}$ 345 signaling (Funai et al. 2016 and Park et al. 2015). Therefore, the activation of AMPK (Merlin et 346 al. 2010) by choline supplementation improved the metabolic condition of Pcyt $2^{+/-}$muscle and 347 indicated that choline had exercise mimetic properties for IR muscle. The question remains if 348 muscle $\mathrm{Ca}^{2+}$ signaling is also activated by choline supplementation and will require further 349 investigation.

The general role of AMPK is to stimulate FA oxidation and to diminish TAG synthesis by lipogenesis, and they are both in effect in choline supplemented Pcyt $2^{+/-}$muscle. Deactivation 352 of ACC with choline treatment is beneficial because ACC catalyzes the formation of malonyl353 CoA, the rate limiting step in FA biosynthesis. Additionally, choline increased the expression of 354 the mitochondrial regulators, Ppar $\alpha, \operatorname{Pgc} 1 \alpha$ and Cpt1. Ppar $\alpha$ stimulates FA oxidation and Pgc1 $\alpha$ 355 regulates mitochondrial biogenesis, while Cpt1 is the rate limiting step in FA transport in the 356 mitochondria.

Choline supplemented Pcyt $2^{+/-}$muscle demonstrated improvements in insulin signaling.

358 There was an increase in IRS1, an increase in $\mathrm{p}^{473} \mathrm{Akt}$ (mTORC2 site) and increased muscle 359 glycogen content with choline treatment. Glycogen synthesis and content are typically decreased 360 in IR. This occurs because elevated GSK3 phosphorylation leads to the inhibition of glycogen 361 synthase activity (Liu et al. 2015). Furthermore, GSK phosphorylates Raptor, an mTORC1 362 associated scaffold protein, which is important in mTORC1 activation (Stretton et al. 2015). 
363 mTORC1 was hyperactivated in $\mathrm{Pcyt}^{+/-}$muscle and there was a decrease in activation with

364 choline treatment. mTORC1 is a regulator of protein synthesis (Wullschleger et al. 2006), and 365 the activation of this protein was normalized with choline treatment. AMPK activation has been 366 shown to inhibit the activation of mTORC1 (Hong-Brown et al. 2010), and is likely most 367 responsible for the normalization of protein turnover in choline supplemented Pcyt $2^{+/-}$muscle. 368 Why was mTORC1 more active in IR Pcyt $2^{+/-}$muscle? Chronic mTORC1 activation has been 369 shown to promote insulin resistance via S6K-dependent phosphorylation of IRS1 leading to its 370 degradation and the inhibition of insulin signalling pathway (Um et al. 2004). Typically, 371 mTORC1 is activated via Akt phosphorylation but this is not the case in IR muscle. mTORC1

372 hyperactivation was likely a consequence of increased nutrient availability/elevated proteolysis 373 in IR Pcyt $2^{+/-}$muscle, and choline supplementation restored this balance (Bond 2016).

375 Conclusion

Choline supplementation has the ability to affect muscle metabolism and improve insulin

377 signaling in mice that have a reduced proficiency to synthesize the membrane phospholipid PE.

378 The mechanisms that link choline with improvements in IR muscle signaling and energy

379 metabolism involve multiple parameters. These include the normalization of gene expression to 380 favour less FA formation and more FA oxidation, causing depression of lipogenesis, increases in 381 muscle glycogen and activation of AMPK. These data suggest that choline supplemented in the 382 diet could normalize muscle FA and TAG homeostasis in order to diminish the IR displayed by 383 perturbed PE biosynthesis. 
384 Acknowledgements

385 This work was supported by an Ontario Graduate Scholarship (AT) and an operating grant (MB)

386 and USRA award (MY) from the National Sciences and Engineering Research Council of 387 Canada.

388

389 Competing interests

390 The authors have declared that no competing interests exist.

391

392 Author contributions

393 Conceived and designed the experiments: AT and LCS. Performed the experiments: AT, 394 MY. Analyzed the data: AT, MB. Wrote the paper: AT, MB. 
395

396

397

398

399

400

401

402

403

404

405

406

$407 \quad 93$

408 Cordero P, Campion J, Milagro FI, Martinez JA. 2013. Transcriptomic and epigenetic changes in 409 early liver steatosis associated to obesity: Effect of dietary methyl donor supplementation. Mol 410 Genet Metab. 110:388-95.

411 Detopoulou P, Panagiotakos DB, Antonopoulou S, Pitsavos C, Stefanadis C. 2008. Dietary

412 choline and betaine intakes in relation to concentrations of inflammatory markers in healthy 413 adults: the ATTICA study. Am J Clin Nutr. 87:424-30.

414 Ejaz A, Martinez-Guino L, Goldfine AB, Ribas-Aulinas F, De Nigris V, Ribo S, Gonzalez415 Franquesa A, Garcia-Roves PM, Li E, Dreyfuss JM, Gall W, Kim JK, Bottiglieri T, Villarroya F, 
416 Gerszten RE, Patti ME, Lerin C. 2016. Dietary Betaine Supplementation Increases Fgf21 Levels

417 to Improve Glucose Homeostasis and Reduce Hepatic Lipid Accumulation in Mice. Diabetes

$418 \quad$ 65:902-12.

419 Folch J, Lees M, Sloane-Stanley GH. 1957. A simple method for the isolation and purification of 420 total lipids from animal tissues. J Biol Chem. 226:497-509.

421 Fullerton MD, Hakimuddin F, Bakovic M. 2007. Developmental and Metabolic Effects of 422 Disruption of the Mouse CTP:Phosphoethanolamine Cytidylyltransferase Gene (Pcyt2). Mol Cell 423 Biol. 27:3327-36.

424 Fullerton MD, Hakimuddin F, Bonen A, Bakovic M. 2009. The development of a metabolic 425 disease phenotype in CTP:phosphoethanolamine cytidylyltransferase-deficient mice. J Biol 426 Chem. 284:25704-13.

427 Funai K, Lodhi IJ, Spears LD, Yin L, Song H, Klein S, Semenkovich CF. 2016. Skeletal Muscle 428 Phospholipid Metabolism Regulates Insulin Sensitivity and Contractile Function. Diabetes. $429 \quad 65: 358-70$.

430 Gao X, Wang Y,Randell E, Pedram P, Yi Y, Gulliver W' Sun G. 2016. Higher Dietary Choline 431 and Betaine Intakes Are Associated with Better Body Composition in the Adult Population of 432 Newfoundland, Canada. PLoS One 11(5):e015540.

433 Haga T. 1971. Synthesis and release of $\left[{ }^{14} \mathrm{C}\right]$-acetylcholine in synaptosomes. J Neurochem. $434 \quad 18: 781-98$. 
435 Hong-Brown LQ, Brown CR, Kazi AA, Huber DS, Pruznak AM, Lang CH. 2010. Alcohol and 436 PRAS40 knockdown decrease mTOR activity and protein synthesis via AMPK signaling and 437 changes in mTORC1 interaction. J Cell Biochem. 109:1172-1184.

438 Kase ET, Feng YZ, Badin PM, Bakke SS, Laurens C, Coue M, Langin D, Gaster M, Thoresen 439 GH, Rustan AC, Moro C. 2015. Primary defects in lipolysis and insulin action in skeletal muscle 440 cells from type 2 diabetic individuals. Biochim Biophys Acta. 1851:1194-1201.

441 Koh HJ, Brandauer J, Goodyear LJ. 2008. LKB1 and AMPK and the regulation of skeletal 442 muscle metabolism. Curr Opin Clin Nutr Metab Care. 11:227-32.

443 Liu TY, Shi CX, Gao R, Sun HJ, Xiong XQ, Ding L, Chen Q, Li YH, Wang JJ, Kang YM, Zhu 444 GQ. 2015. Irisin inhibits hepatic gluconeogenesis and increases glycogen synthesis via the $445 \mathrm{PI}$ K/Akt pathway in type 2 diabetic mice and hepatocytes. Clin Sci. 129:839-50.

446 Liu X, Yuan Y, Niu Y, Niu W, Fu L. 2012. The role of AMPK/mTOR/S6K1 signaling axis in 447 mediating the physiological process of exercise-induced insulin sensitization in skeletal muscle 448 of C57BL/6 mice. Biochim Biophys Acta. 1822:1716-26.

449 Lo S, Russell JC, Taylor AW. 1970. Determination of glycogen in small tissue samples. J Appl 450 Physiol. 28:234-6.

451 Merlin J, Evans BA, Csikasz RI, Bengtsson T, Summers RJ, Hutchinson DS. 2010. The M3452 muscarinic acetylcholine receptor stimulates glucose uptake in L6 skeletal muscle cells by a 453 CaMKK-AMPK-dependent mechanism. Cell Signal. 22:1104-13.

454 Michel V, Bakovic M. 2012. The Ubiquitous Choline Transporter SLC44A1. Cent Nerv Syst 455 Agents Med Chem. 12:70-81. 
456 Michel V, Singh RK, Bakovic M. 2011. The impact of choline availability on muscle lipid

457 metabolism. Food Funct. 2:53-62.

458 Mitsuhashi S, Hatakeyama H, Karahashi M, Koumura T, Nonaka I, Hayashi YK, Noguchi S, 459 Sher RB, Nakagawa Y, Manfredi G, Goto Y, Cox GA, Nishino I. 2011. Muscle choline kinase 460 beta defect causes mitochondrial dysfunction and increased mitophagy. Hum Mol Genet. $461 \quad 20: 3841-51$.

462 Nassenstein C, Wiegand S, Lips KS, Li G, Klein J, Kummer W. 2015. Cholinergic activation of 463 the murine trachealis muscle via non-vesicular acetylcholine release involving low-affinity 464 choline transporters. Int Immunopharmacol. 29:173-80.

465 Niculescu MD. 2013. Choline and Phosphatidylcholine. Encyclopedia of Human Nutrition. $466 \quad 1: 346-351$.

467 Park DR, Park KH, Kim BJ, Yoon CS, Kim UH. 2015. Exercise ameliorates insulin resistance 468 via $\mathrm{Ca} 2+$ signals distinct from those of insulin for GLUT4 translocation in skeletal muscles. 469 Diabetes. 64:1224-34.

470 Penry JT, Manore MM. 2008. Choline: an important micronutrient for maximal endurance471 exercise performance? Int J Sport Nutr Exerc Metab. 18:191-203.

472 Purohit V, Abdelmalek MF, Barve S, Benevenga NJ, Halsted CH, Kaplowitz N, Kharbanda KK, 473 Liu QY, Lu SC, McClain CJ, Swanson C, Zakhari S. 2007. Role of S-adenosylmethionine, 474 folate, and betaine in the treatment of alcoholic liver disease: summary of a symposium. Am J 475 Clin Nutr. 86:14-24.

476 Sachan DS, Hongu N, Johnsen M. 2005. Decreasing oxidative stress with choline and carnitine 477 in women J Am Coll Nutr. 24:172-6. 
478 Schenkel LC, Bakovic M. 2014. Palmitic acid and oleic acid differentially regulate choline 479 transporter-like 1 levels and glycerolipid metabolism in skeletal muscle cells. Lipids. 49:731-44.

480 Schenkel LC, Sivanesan S, Zhang J, Wuyts B, Taylor A, Verbrugghe A, Bakovic M. 2015. 481 Choline supplementation restores substrate balance and alleviates complications of Pcyt2 482 deficiency. J Nutr Biochem. 26:1221-34.

483 Schmitz-Peiffer C. 2002. Protein kinase C and lipid-induced insulin resistance in skeletal muscle. 484 Ann N Y Acad Sci. 967:146-57.

485 Selathurai A, Kowalski GM, Burch ML, Sepulveda P, Risis S, Lee-Young RS, Lamon S, Meikle 486 PJ, Genders AJ, McGee SL, Watt MJ, Russell AP, Frank M, Jackowski S, Febbraio MA, Bruce 487 CR. 2015. The CDP-Ethanolamine Pathway Regulates Skeletal Muscle Diacylglycerol Content 488 and Mitochondrial Biogenesis without Altering Insulin Sensitivity. Cell Metab. 21:718-30.

489 Senesi P, Luzi L, Montesano A, Mazzocchi N, Terruzzi I. 2013. Betaine supplement enhances 490 skeletal muscle differentiation in murine myoblasts via IGF-1 signaling activation. J Transl Med. $491 \quad$ 11:1-12

492 Sher RB, Aoyama C, Huebsch KA, Ji S, Kerner J, Yang Y, Frankel WN, Hoppel CL, Wood PA, 493 Vance DE, Cox GA. 2006. A rostrocaudal muscular dystrophy caused by a defect in choline 494 kinase beta, the first enzyme in phosphatidylcholine biosynthesis. J. Biol. Chem. 281: 4938-48.

495 Stretton C, Hoffmann TM, Munson MJ, Prescott A, Taylor PM, Ganley IG, Hundal HS. 2015. 496 GSK3-mediated raptor phosphorylation supports amino-acid dependent mTORC1-directed 497 signalling. Biochem J. 470:207-21. 
498 Tryndyak VP, Marrone AK, Latendresse JR, Muskhelishvili L, Beland FA, Pogribny IP. 2016. 499 MicroRNA changes, activation of progenitor cells and severity of liver injury in mice induced by 500 choline and folate deficiency. J Nutr Biochem. 28:83-90.

501 Um SH, Frigerio F, Watanabe M, Picard F, Joaquin M, Sticker M, Fumigalli S, Allegrini PR, 502 Kozma SC, Auwerx J, Thomas G. 2004. Absence of S6K1 protects against age- and diet-induced 503 obesity while enhancing insulin sensitivity. Nature. 431:200-5.

504 Wullschleger S, Loewith R, Hall MN. 2006. TOR signaling in growth and metabolism Cell. 505 124:471-484.

506 Yamada H, Imajoh-Ohmi S, Haga T. 2012. The high-affinity choline transporter CHT1 is 507 regulated by the ubiquitin ligase Nedd4-2. Biomed Res. 33:1-8.

508 Yuan Z, Tie A, Tarnopolsky M, Bakovic M. 2006. Genomic organization, promoter activity, and 509 expression of the human choline transporter-like protein 1. Physiol Genomics. 26:76-90.

510 Yuan Z, Wagner L, Poloumienko A, Bakovic M. 2004. Identification and expression of a mouse 511 muscle-specific CTL1 gene. Gene. 341: 305-12.

512 Zeisel SH, da Costa KA. 2009. Choline: an essential nutrient for public health. Nutr Rev. 67:61551323.

514 Zeisel SH. 2012. Dietary choline deficiency causes DNA strand breaks and alters epigenetic 515 marks on DNA and histones. Mutat Res. 733: 34-8. 
518 Table 1: Primer sequences, fragment size and melting temperature for the amplification of genes 519 involved in lipolysis, lipogenesis, mitochondrial function and phospholipid synthesis.

\begin{tabular}{|c|c|c|c|c|}
\hline Gene & Forward Primer 5'-3' & Reverse Primer 5'-3' & $\begin{array}{l}\mathrm{T}_{\mathrm{M}} \\
\left({ }^{\mathrm{O}} \mathrm{C}\right)\end{array}$ & $\begin{array}{l}\text { Size } \\
\text { (bp) }\end{array}$ \\
\hline Gapdh & ACCACAGTCCATGCCATCAC & TCCACCACCCTGTTGCTGTA & 57 & 452 \\
\hline Pss2 & GAGTGGCTGTCCCTGAAGAC & TCGTAGATCTCACGCATGGC & 59 & 305 \\
\hline Atgl & CAACGCCACTCACATCTACGG & GGACACCTCAATAATGTTGGCAC & 57 & 106 \\
\hline Hsl & ACGCTACACAAAGGCTGCTT & TCGTTGCGTTTGTAGTGCTC & 59 & 125 \\
\hline Lpl & GCTCGCACGAGCGCTCCATT & CCTCGGGCAGGGTGAAGGGAA & 59 & 351 \\
\hline $\operatorname{Pgc} 1 \alpha$ & TTGACTGGCGTCATTCGGG & GAAGGACTGGCCTCGTTGTC & 59.5 & 396 \\
\hline Fas & AGATGGAAGGCTGGGCTCTA & GAAGCGTCTCGGGATCTCTG & 59 & 268 \\
\hline Ctl1 & GAACGCTCTGCGAGTGGCTGC & CGGCTTTAGCTCTCGGGCGT & 49 & 376 \\
\hline Pcyt1 & ATGCACAGAGAGTTCAGCTAAAG & GGGCTTACTAAAGTCAACTTCAA & 50 & 170 \\
\hline Pss1 & CTGTTGTGCAATGGTGGTGG & GGCTGGCTTGGAACACAAAG & 59.5 & 283 \\
\hline Psd & GTTTGCTGTCACGTGCCTGTG & CAGTGCAAGCCACATACGGG & 59.5 & 205 \\
\hline Dgat2 & GGCGCTACTTCCGAGACTAC & TCTTTAGGGTGACTGCGTTC & 58.5 & 255 \\
\hline Scd1 & CGCATCTCTATGGATATCGCCCC & CTCAGCTACTCTTGTGACTCCCG & 57 & 279 \\
\hline Dgat1 & ATCCAGACAACCTGACCTACCG & GACCGCCAGCTTTAAGAGACGC & 57 & 257 \\
\hline Cpt1 & CCCATGTGCTCCTACCAGAT & GGTCTCATCGTCAGGGTTGT & 57 & 396 \\
\hline Ppar $\alpha$ & CGCATGTGAAGGCTGTAAGGGC & GGCTTCGTGGATTCTCTTGCCC & 57 & 289 \\
\hline Srebp1c & TCACAGGTCCAGCAGGTCCC & GGTACTGTGGCCAAGATGGTCC & 57 & 438 \\
\hline Acc & CGCATCTCTATGGATATCGCCCC & CTCAGCTACTCTTGTGACTCCCG & 57 & 295 \\
\hline
\end{tabular}




\section{Figure Legends}

Fig. 1 Choline modifies muscle glycerolipid and glucose metabolism. A. Choline supplementation modified choline-containing phospholipids and cholesterol. The IR Pcyt $2^{+/-}$ muscle contained reduced FC and PI relative to the control Pcyt $2^{+/+}$muscle. CE and PE were not significantly modified in Pcyt $2^{+/-}$muscle with respect to the other two groups. Pcyt $2^{+/-} \mathrm{CHO}$ muscle had decreased FA content and increased PC and SM content with respect to Pcyt $2^{+/-}$ muscle. (B) The relative phospholipid ratio of PC/PE increased with choline supplementation, and (C) the relative SM/FC ratio was not affected with choline treatment and remained increased as in Pcyt $2^{+/-}$muscle. D. and E. Choline reduced muscle TAG and DAG. Total TAG content (D) and both relative TAG and DAG ratios $(E)$ were decreased with choline supplementation

$\left(P c y t 2^{+/-} \mathrm{CHO}\right)$ relative to Pcyt $2^{+/-}$and they were similar to the control Pcyt $2^{+/+}$mice. F. Choline increases muscle glycogen content. Pcyt $2^{+/-}$mice have dramatically reduced glycogen content that was increased with choline treatment, approaching the value of the Pcyt $2^{+/+}$mice. (A-F) number of mice per each group: $n=4$; one-way ANOVA: ${ }^{*} \mathrm{p}<0.05$ compared to Pcyt $2^{+/+}$and \#p $<0.05$ compared to Pcyt $2^{+/-}$.

\section{Fig. 2 Choline regulates expression of genes involved in muscle fatty acid and triglyceride} metabolism. A. The phospholipid biosynthetic genes are not significantly altered across groups. Ctl1, Pcyt1 and Psd were unmodified while Pss 1 and Pss2 expression were modestly decreased with choline treatment with respect to Pcyt $2^{+/+}$and Pcyt $2^{+/-}$muscle. B. Choline normalizes the expression of genes involved in lipogenesis. (a) The expression of FA biosynthetic genes Scdl, Fas and Srebplc were elevated in IR Pcyt $2^{+/-}$muscle and choline normalized their levels; (b) Choline also normalized the expression of genes involved in TAG synthesis. The expression of Dgat1 and Dgat 2 were elevated in Pcyt $2^{+/-}$relative to Pcyt $2^{+/+}$muscle and became decreased with choline treatment. C. Choline has no major effect on muscle lipolysis. The expression of Lpl and Atgl were modestly increased in Pcyt $2^{+/-}$muscle relative to the wildtype Pcyt $2^{+/+}$muscle but not affected by choline treatment. The expression of Hsl that was slightly reduced in Pcyt $2^{+/-}$muscle 
550

551

552

553

554

555

556

557

558

559

560

561

562

563

564

565

566

567

568

569

570

571

572

573

574

575

576

577

was increased with choline treatment. The intensity of bands were normalized to Gapdh gene control and expressed in arbitrary units; (A-C) number of mice per group: $n=4$; one-way ANOVA: $* \mathrm{p}<0.05$ compared to Pcyt $2^{+/+}$and \#p $<0.05$ compared to Pcyt $2^{+/}$.

Fig. 3 Choline improves mitochondrial energy production. AMPK activation (A) and ACC inhibition $(B)$ with choline. Pcyt $2^{+/-}$muscle showed a dramatic reduction in AMPK and ACC phosphorylation (pAMPK:AMPK and pACC:ACC ratio) and choline treatment (Pcyt $2^{+/-} \mathrm{CHO}$ muscle) increased the phosphorylation of both species. C. The amounts of AMPK, pAMPK, ACC and pACC proteins was assessed using $\beta$-tubulin as a control. The intensity of bands was quantified by densitometry and expressed in arbitrary units. ${ }^{*} p<0.05$ compared to Pcyt $2^{+/+}$and $\# \mathrm{p}<0.05$ compared to Pcyt $2^{+/-}$D. Mitochondrial gene activation with choline. The expression of Cpt1, Ppar $\alpha$ and Pgc1 $\alpha$ was increased with choline treatment whereas the expression of Acc was unchanged. The intensity of PCR bands were quantified using ImageJ software and expressed as arbitrary units. (A-C) Each experiment was repeated 3 times with $n=3$ per group. D. The number of mice per group: $\mathrm{n}=4$; one-way ANOVA $\mathrm{n}=4$ per group). ${ }^{*} \mathrm{p}<0.05$ compared to Pcyt $2^{+/+}$and \#p $<0.05$ compared to Pcyt $2^{+/-}$.

Fig. 4 Choline reduces $\mathrm{PKC \alpha}$ and restores muscle insulin signaling. A. Representative immunoblots showing differences in insulin signalling pathway. The amounts of IRS1, PI3K, Akt, pAkt and PKC $\alpha$ proteins were assessed using $\beta$-tubulin as a control.. B. IRS1 protein was diminished in Pcyt $2^{+/-}$muscle and completely restored with choline treatment. C. PI3K levels were elevated in Pcyt $2^{+/-}$muscle and decreased with choline treatments. D. pThr ${ }^{308} \mathrm{Akt}$ :Akt ratio was unchanged across all groups whereas (E.) muscle pSer ${ }^{473} \mathrm{Akt}$ :Akt increased with choline treatment relative to $\mathrm{Pcyt} 2^{+/-}$muscle. F. PKC $\alpha$ that was elevated in IR Pcyt2 ${ }^{+/-}$muscle dramatically decreased with choline treatments. The intensity of bands were normalized relative to $\beta$-tubulin and expressed in arbitrary units relative to the normal Pcyt $2^{+/+}$muscle. (A-F) Each experiment was repeated 3 times with $n=3$ per group; one-way ANOVA: $* \mathrm{p}<0.05$ compared to Pcyt $2^{+/+}$and \#p $<0.05$ compared to Pcyt $2^{+/-}$. 
578 Fig. 5 Choline reduces mTORC1 activity and stimulates autophagy. A. Representative 579 immunoblots for mTORC1, $\mathrm{p}^{2448}$ mTORC1, LC3I, LC3II, CTL1, and $\beta$ tubulin in choline treated 580 and untreated groups. B. Choline reduces muscle mTORC1 activation. Total mTORC1 content 581 was unchanged across groups but $\mathrm{p}^{2448}$ mTORC:mTORC1 ratio was decreased with choline 582 treatment in relation to $\mathrm{Pcyt}^{+/-}$C. Choline increases muscle autophagy. Choline treatment 583 increased Pcyt $2^{+/-}$LC3 lipidation shown as elevated LC3II:LC3I ratio. D. Choline restores the 584 amount of choline transporter CTL1. CTL1 protein was diminished in Pcyt $2^{+/-}$muscle but 585 became restored with choline treatment. The intensity of all protein bands and control $\beta$ tubulin 586 were quantified by densitometry and expressed as arbitrary units (fold change) relative to wild 587 type muscle. (A-D): Each experiment was repeated 3 times ( $\mathrm{n}=3$ per group); one-way ANOVA: $588 * \mathrm{p}<0.05$ compared to $\mathrm{Pcyt} 2^{+/+}$and \#p $<0.05$ compared to Pcyt $2^{+/-}$. 


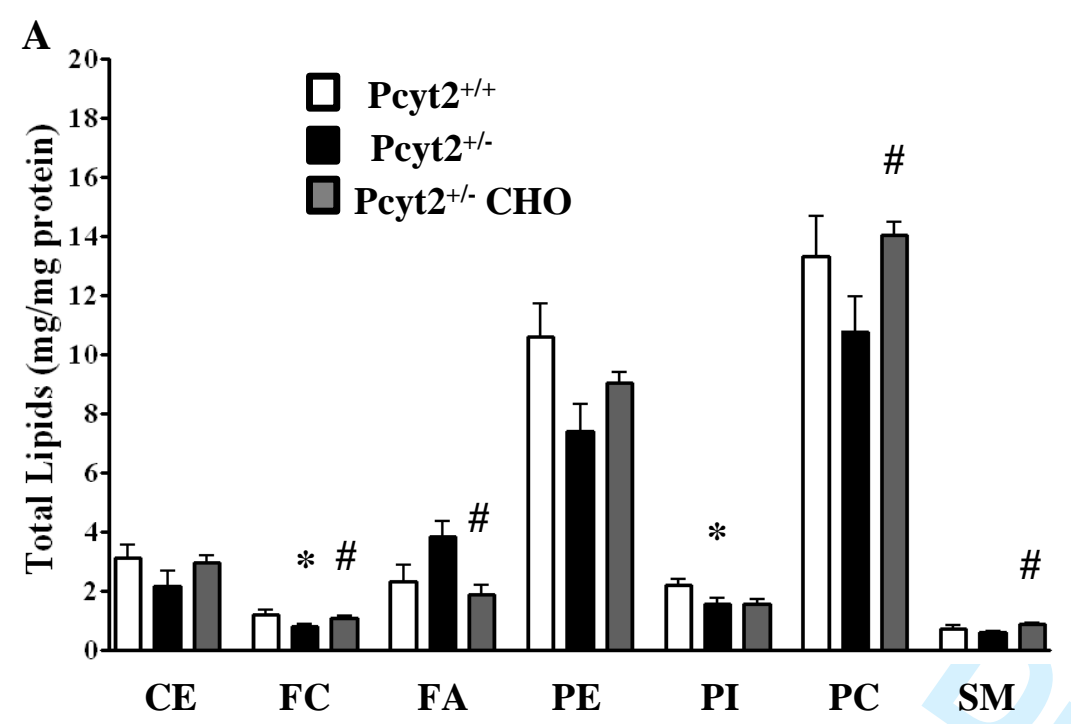

B

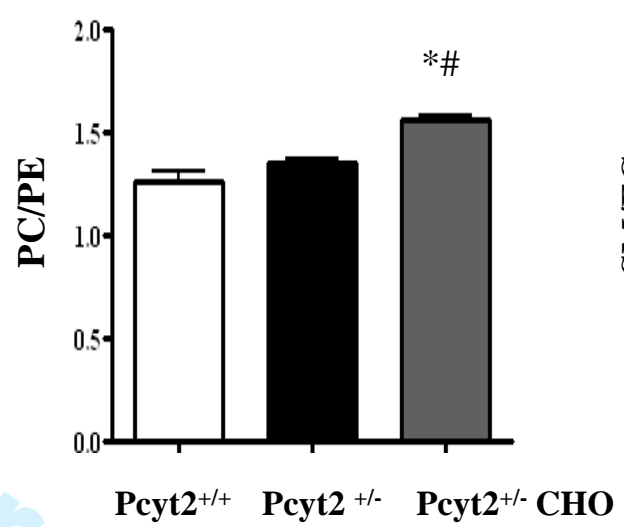

C

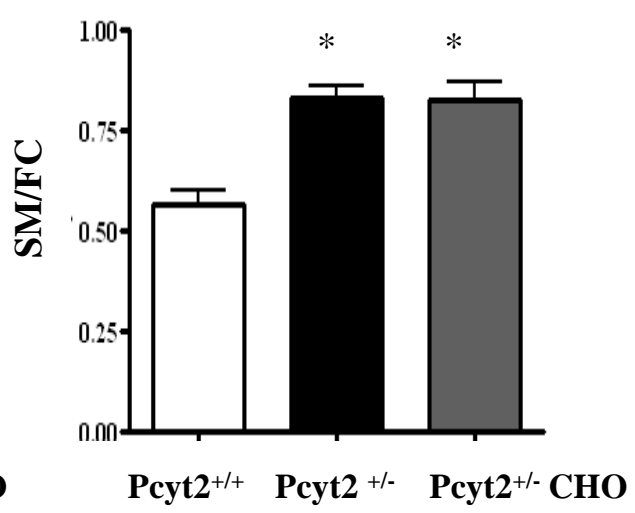

D

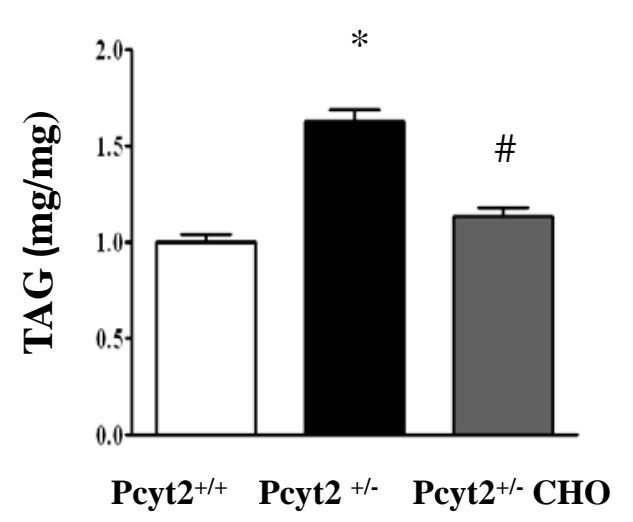

E

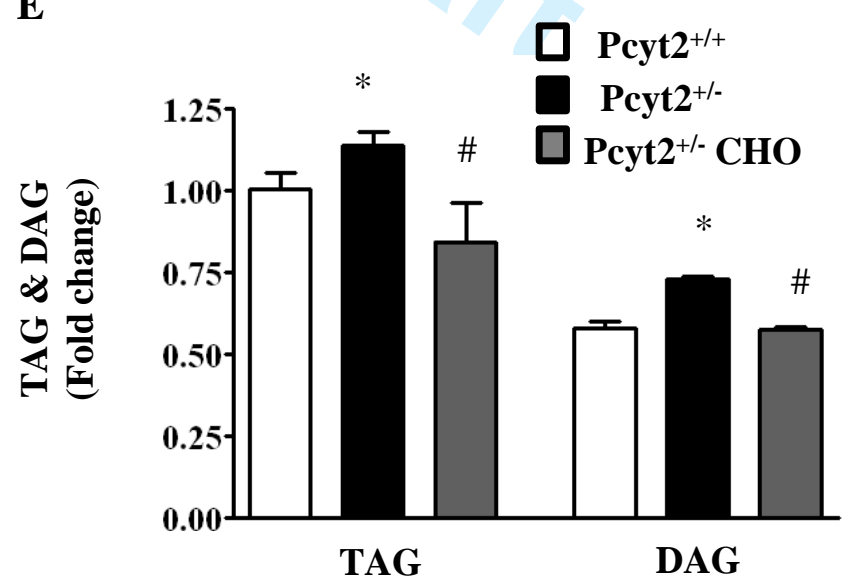

F

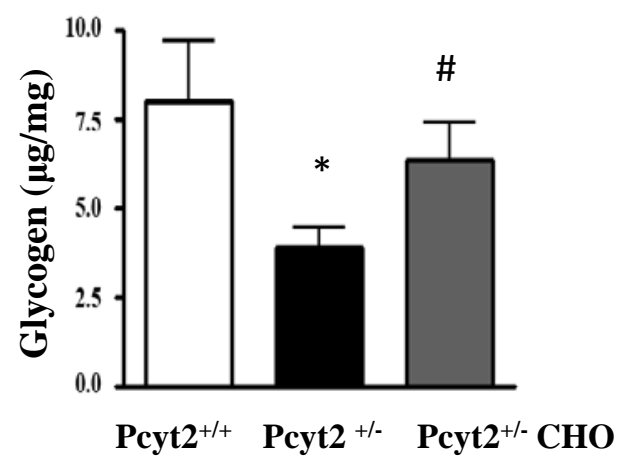



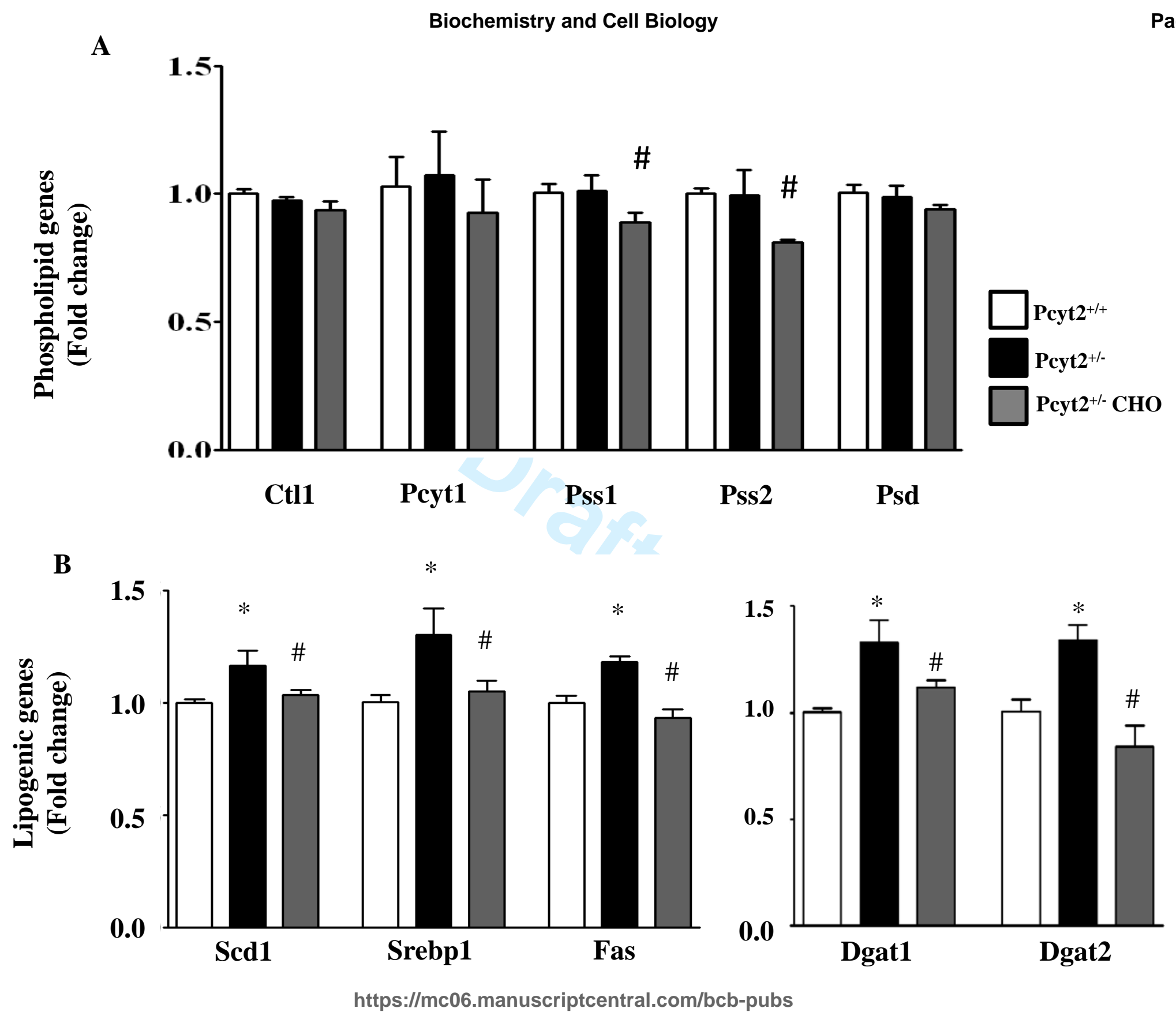

Figure $2 A \& B$ 


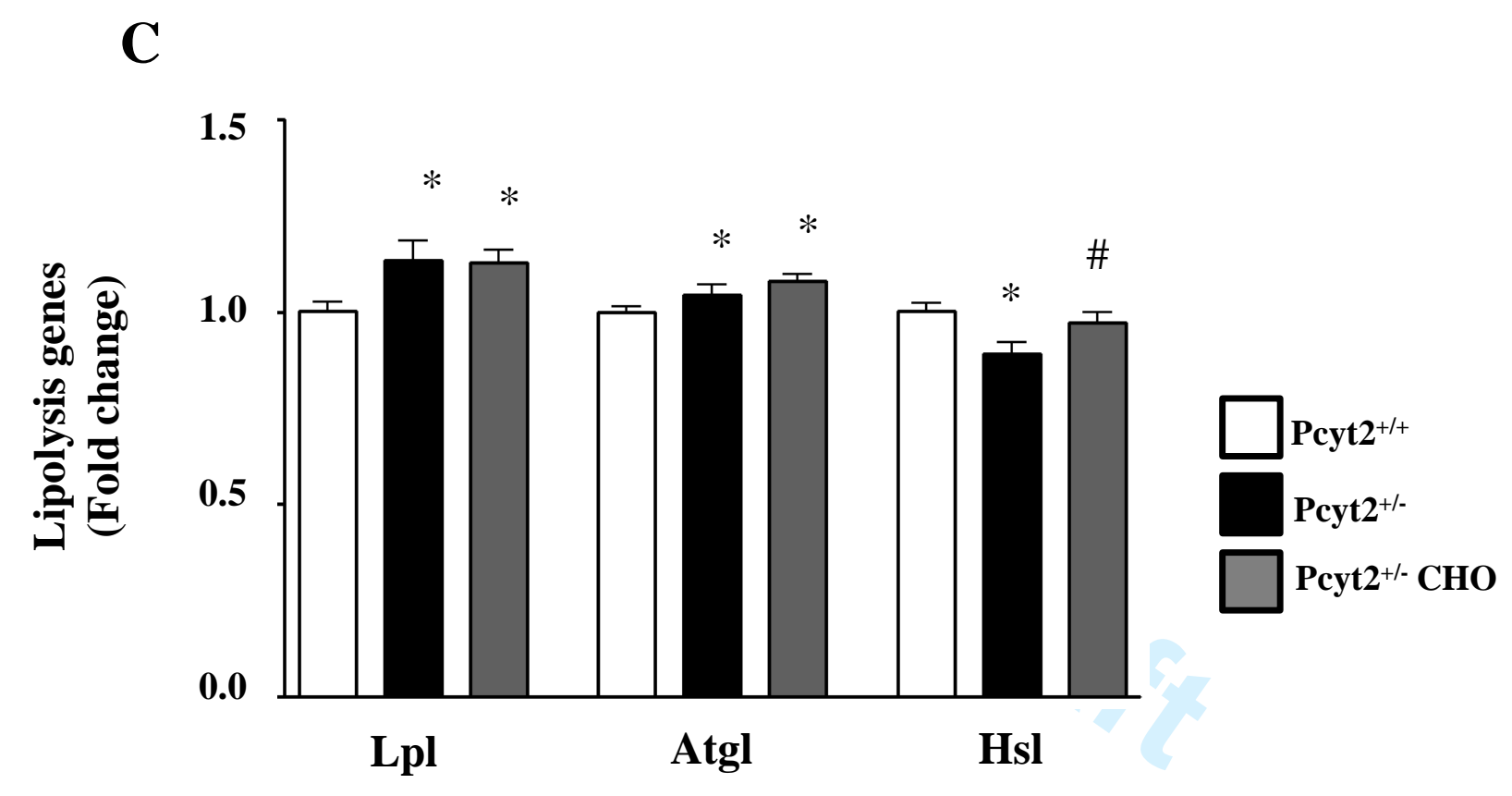

Figure 2C 
A

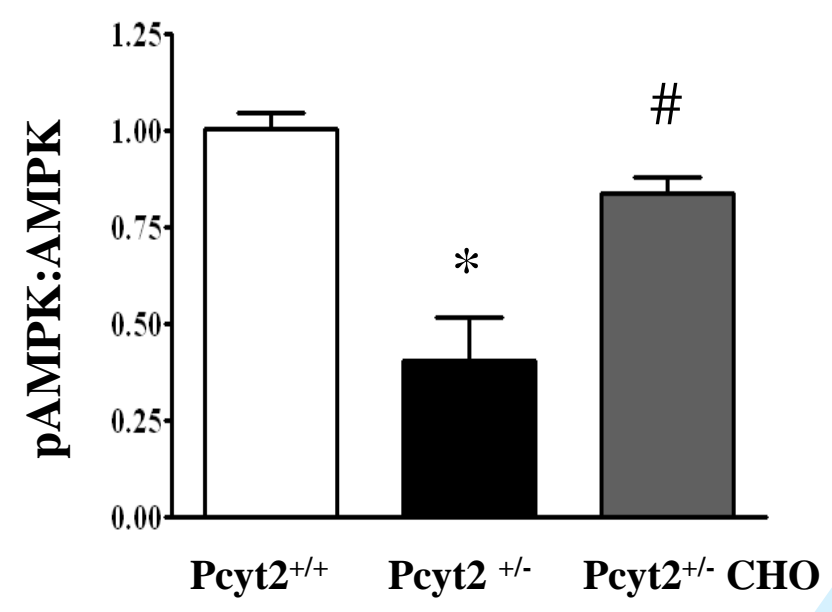

B

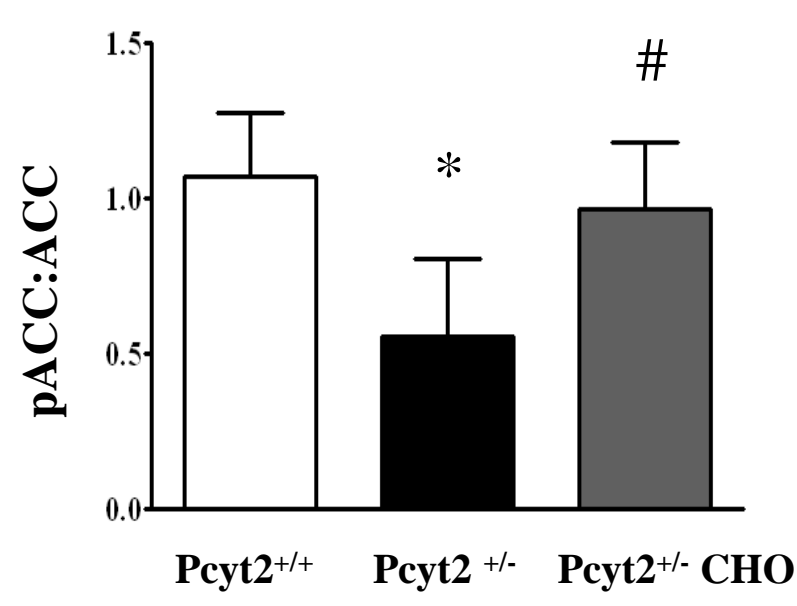

C

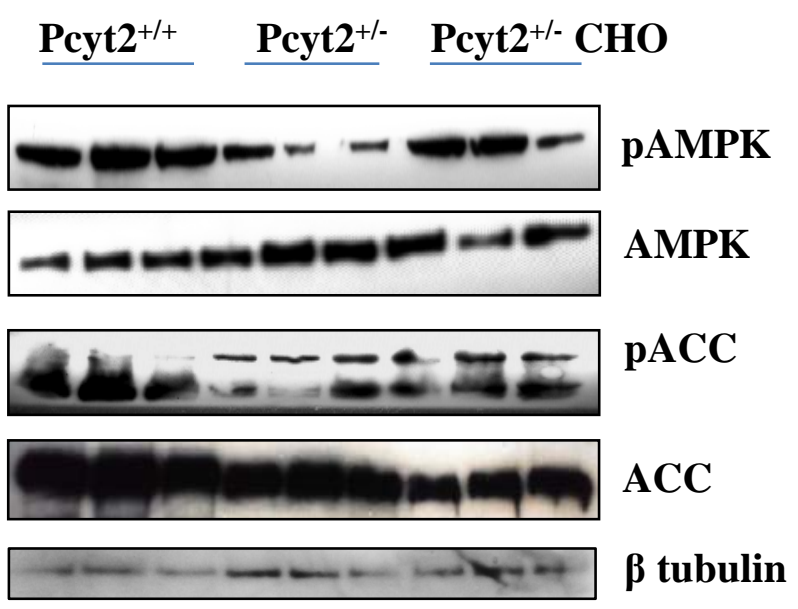

D

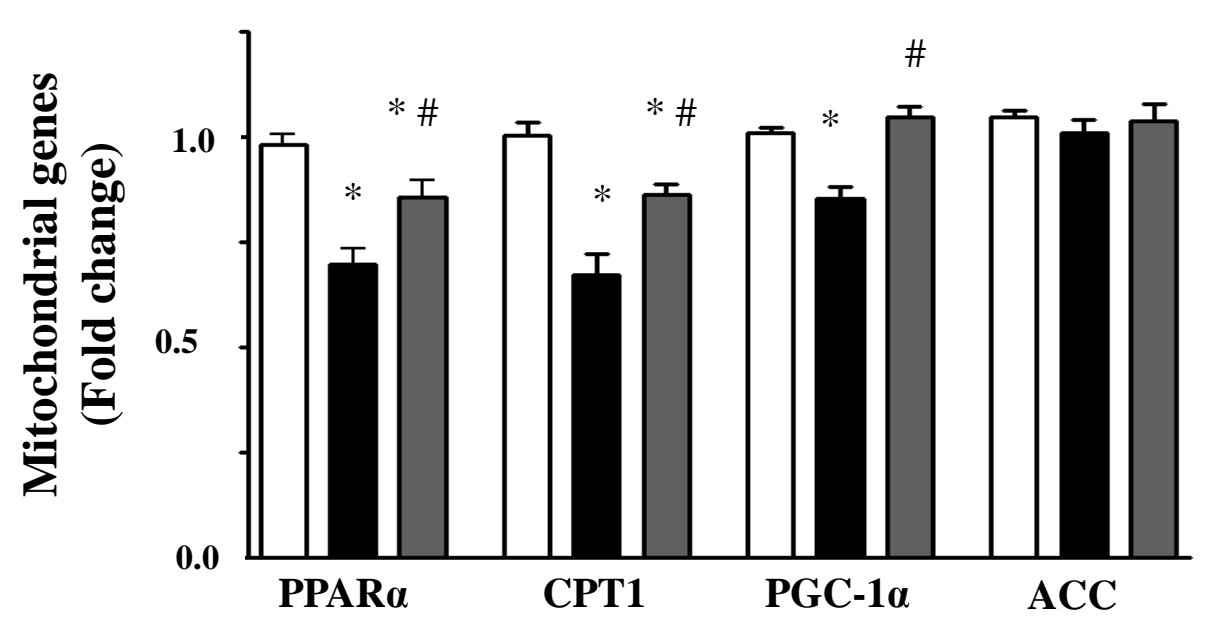

Figure 3 https://mc06.manuscriptcentral.com/bcb-pubs 
A Pcyt2 $^{+/+}$Pcyt2 $^{+/-}$Pcyt2 $^{+/}$CHO
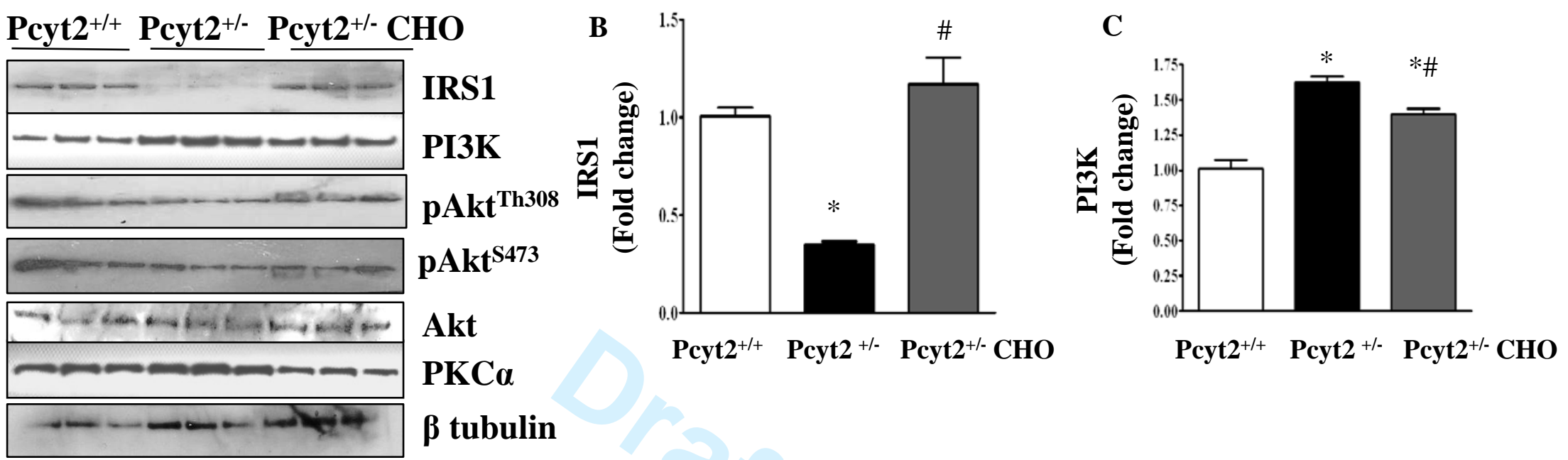

D

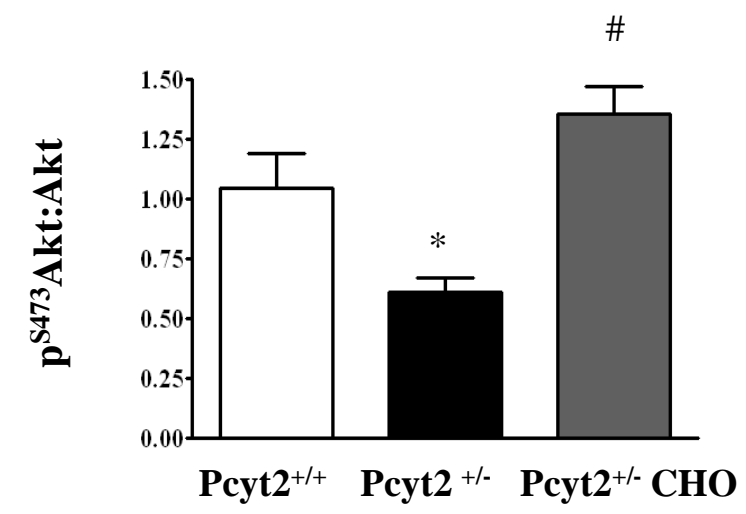

$\mathbf{E}$

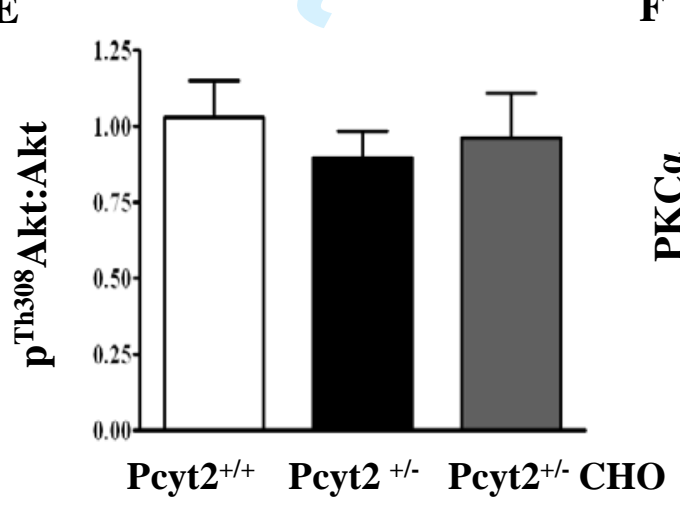

$\mathbf{F}$

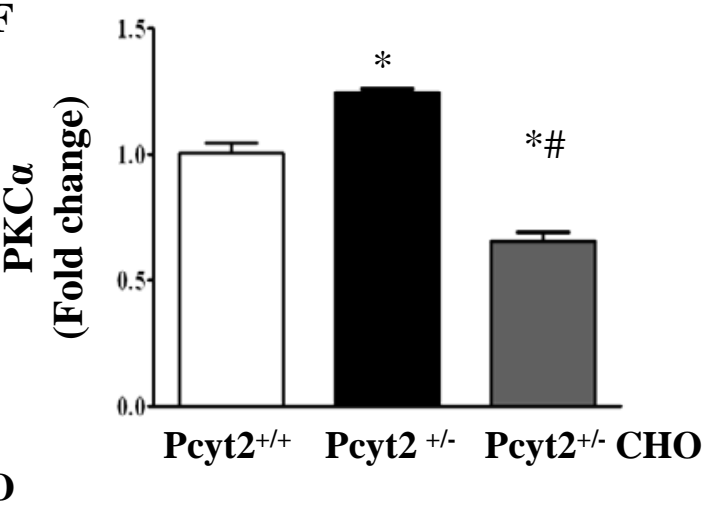




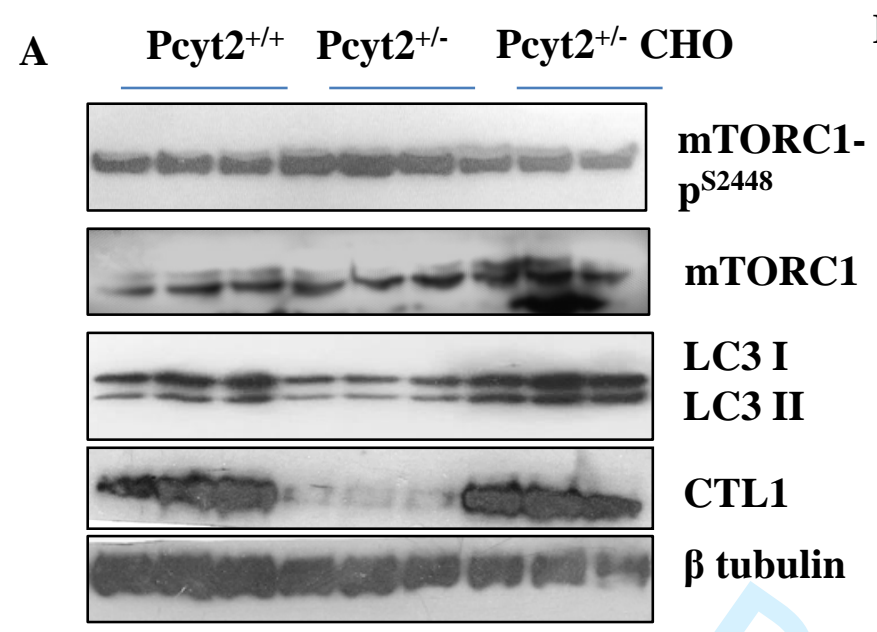

B

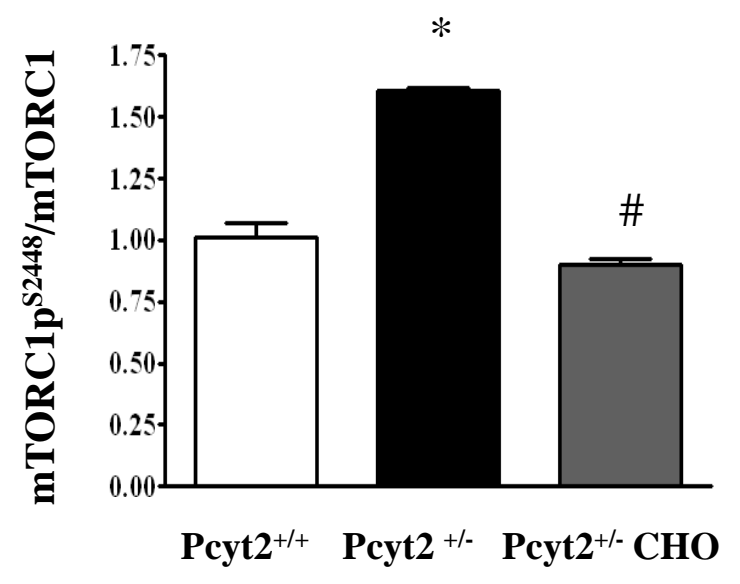

C

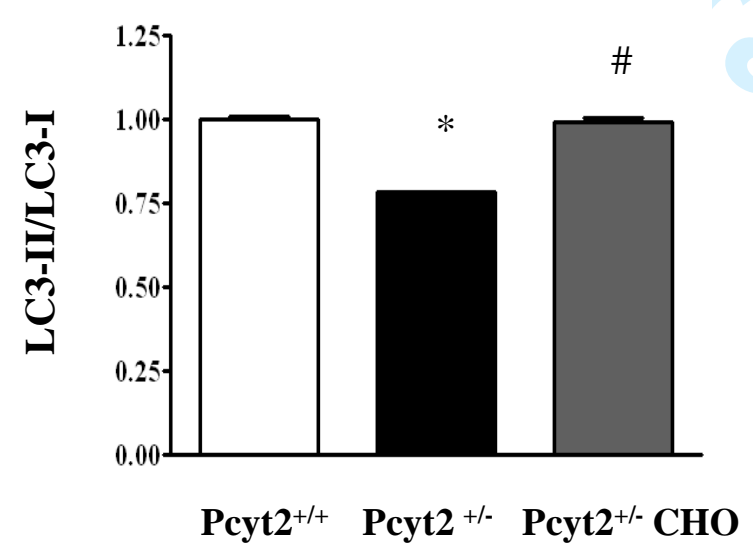

D

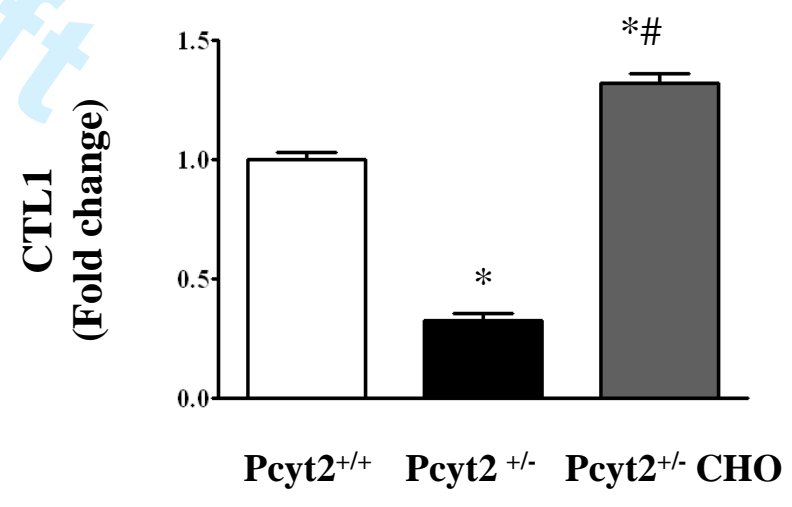

